\title{
Leaving Against Medical Advice Among Patients With Brain Tumours in the Middle East: Khoula Hospital Experience
}

\author{
Tariq AL-Saadi ${ }^{1,2}{ }^{*}$, Ali ALSharqi ${ }^{3}$, Jawahir ALSharqi ${ }^{4}$, Khadija ALBulushi ${ }^{4}$, Asma ALRasbi ${ }^{3}$, Maather ALFarsi ${ }^{3}$, \\ Shamsa ALGhafri ${ }^{3}$, Said Al-Abri ${ }^{5}$, Hatem Al-Saadi ${ }^{6}$
}

${ }^{1}$ Department of Neurosurgery, Montreal Neurological Institute and Hospital - McGill University, Montreal, Canada.

${ }^{2}$ Department of Neurosurgery, Khoula Hospital, Muscat, Sultanate of Oman.

${ }^{3}$ College of Medicine, Sultan Qaboos University, Muscat, Sultanate of Oman.

${ }^{4}$ Pediatric General Foundation Program, Oman Medical Specialty Board, Muscat, Sultanate of Oman.

${ }^{5}$ Statistical Researcher, Center for Government Communications, General Secretariat to the Cabinet, Muscat, Sultanate of Oman.

${ }^{6}$ Gulf Research Collaboration Group (GRCG) - North Midlands Institute of Metabolic and Bariatric Surgery, Stafford, United Kingdom.

\begin{abstract}
Background: Leaving against medical advice (LAMA) can be defined broadly as a patient's insistence upon leaving the hospital against the treating team has expressed advice, which is both a challenge and concern for physicians, as these patients lost to follow-up, and their outcomes remain unknown. There is no previous study conducted to find the prevalence and causes of LAMA in brain tumors patients in the Middle East to the best of our knowledge.

Methods: Patients studied in this research are those who were diagnosed with any type of brain tumors and were admitted to the Neurosurgical Department in Khoula Hospital (KH) but signed LAMA in the two years between January 2017 to December 2018 by going through the electronic medical records. Data obtained from the health information system includes socioeconomic characteristics, health status-related data, diagnosis-related data, and the reasons for LAMA

Results: A total number of 302 patients with brain tumors included in this study. Twenty-eight patients $(9.2 \%)$ signed LAMA with a majority of those who signed LAMA were in the young adult's group (3-39 years) and represented 18 (64\%). Eight patients $(28.57 \%)$ among the LAMA group and 43 patients $(15.69 \%)$ in the non-LAMA group have tumors in the frontal lobe, which has found to be the most familiar location (29\%). There was a significant relationship between the reason for LAMA and gender $(P=0.020)$.

Conclusion: Younger patients, male, Omani, newly diagnosed tumors, and tumors in the frontal lobe were all risk factors for LAMA. Education and awareness about LAMA recommended in order to avoid readmission and loss of follow up.

Keywords: Tumor, Brain, leaving, Medical Advice, Oman, LAMA
\end{abstract}

*Correspondence to Tariq Al-Saadi,

Department of Neurosurgery, Montreal Neurological Institute and Hospital McGill University, Montreal, Canada and Department of Neurosurgery, Khoula Hospital, Muscat, Sultanate of Oman. Email: t.dhiyab@hotmail.com

Published online 28 September 2020

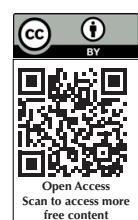

Citation: AL-Saadi T, ALSharqi A, ALSharqi J, ALBulushi K, ALRasbi A, ALFarsi M, ALGhafri S, Al-Abri S, Al-Saadi H. Leaving Against Medical Advice Among Patients With Brain Tumours in the Middle East: Khoula Hospital Experience . Clin Neurosci J. 2020;7(4):179184. doi:10.34172/icnj.2020.22.

\section{Introduction}

Brain and spinal tumors contribute to the main bulk of neurosurgery work in any neurosurgical center. ${ }^{1}$ The term "brain tumors" refers to a mixed group of neoplasms originating from intracranial tissues and the meninges with degrees of malignancy ranging from benign to aggressive. ${ }^{2}$ In Oman, the latest report from the National Oncology Centre and the Ministry of Health $(\mathrm{MoH})$ reveals that 1314 cancer cases registered in 2012, of which $92.2 \%$ were Omani of which leukemia was the most common cancer among children. ${ }^{3}$ A recent study conducted at Khoula Hospital (KH) in Oman showed
1419 patients admitted to the Neurosurgery Department in $2017,{ }^{1}$ of which cranial and spinal tumors accounted for $9.6 \%$. Out of those, thirteen patients singed, leaving against medical advice (LAMA). The study emphasizes the incidence of brain tumors in a sector of the population compared to the data revealed by other studies. ${ }^{1}$ The same study found that meningiomas are making the bulk of it and medulloblastomas remaining the common pediatric tumors. ${ }^{1}$

LAMA can be defined broadly as a patient's insistence upon leaving the hospital against the expressed advice of the treating team. ${ }^{4}$ Patients who leave against medical 
advice are both a challenge and concern for physicians, as these patients have lost to follow-up, and their outcomes remain unknown. ${ }^{5}$ Leaving the hospital against the physician's advice may expose the patient to the risk of inadequately treated medical problems resulting in the need for readmission. ${ }^{6}$ It reflects a failure of the consensus and understanding between the attending physicians and the patient regarding the need for continued hospitalization. ${ }^{7}$ LAMA is not an uncommon request from family members of patients with brain tumors in Oman in the Neurosurgery Department. The phenomenon of LAMA is world-wide, including both rural and urban hospitals. ${ }^{4,5}, 7$ The incidence of LAMA ranges from $>20 \%$ in large urban hospitals, especially alcoholics, drug abusers to $<4 \%$ in small rural hospitals, and medical admissions. ${ }^{7}$ Most of the published data are from retrospective analyses and case-control studies at single urban institutions. ${ }^{6}$ The predictors of LAMA fall within two broad categories; the patient variables which include socio-demographic characteristics, diagnosis, treatment history, behavior; and attitudes toward treatment and the second category is the provider variables including hospital setting and structure, staffing patterns, admission and discharge policies, and physicians' clinical style and experience. ${ }^{8}$

To the best of our knowledge, no previous study has conducted to find the prevalence and causes of LAMA in brain tumor patients in our region. This research aims to study the prevalence and causes of LAMA in the Neurosurgery Department at KH in Oman.

\section{Materials and Methods \\ Study Group and Sample Size}

Patients diagnosed with any type of brain tumor and admitted to the Neurosurgical Department in $\mathrm{KH}$ between January 2017 and December 2018 were identified as the target population. Patients have then categorized into two groups (LAMA group and non-LAMA group). These data obtained from the electronic medical records in the hospital information system (HIS). Inclusion criteria were patients with any brain tumor who left against medical advice, of any nationality, and any age. Patients with extra-cranial tumors (i.e., spinal tumors, skull tumors), who were offered treatment abroad by the treating team or those who were seen only in the clinic and refused admission and treatment excluded from this study.

\section{Data Collection}

A retrospective data collection was conducted by reviewing records available in the hospital's electronic medical records (Health Information System, HIS). These include socioeconomic characteristics (nationality, age, gender, (nationality, age, gender, marital status, health insurance, distance from treating hospital), health status-related data (co-existing comorbidities, history of smoking, alcohol, drug abuse or psychiatric disorders) and diagnosis-related data (time of diagnosis with a brain tumor, location of the tumor, length of hospital stay, the treatment proposed and follow up plan). Reasons for LAMA identified and obtained from the HIS by looking through the medical records for all included patients.

\section{Data Analysis}

The research database was analyzed and processed using the Statistical Package for the Social Sciences (SPSS. v21 Inc, Chicago, Illinois, USA). Continuous variables were expressed as mean \pm SD. Chi-square test was used to obtain the significance of the association between categorized variables, using a $P$-value of $\leq 0.05$ as the cutoff for significance. The ANOVA test was used for variables with a normal distribution pattern to test the significance of differences between the means of the groups. The association between length of hospital stay and reason for LAMA with other variables among LAMA cases was tested with Pearson $\chi^{2}$ test.

\section{Results}

The demographic information of all neurosurgical patients with brain tumors admitted to KH between January 2017 and December 2018 is shown in Table 1. A total number of 302 patients with brain tumors included in this study. Twenty-eight patients signed LAMA with Omani patients accounting for $93 \%(n=26)$. The majority of those who signed LAMA $(64 \%, \mathrm{n}=18)$ were in the young adult group (3-39 years). Among patients in the LAMA group, 19 patients were male, and nine were female. Twentythree cases newly diagnosed with brain cancer, and five were known to have brain cancers diagnosed before that admission. Eight patients (28.57\%) among the LAMA group and 43 patients (15.69\%) in the non-LAMA group have tumors in the frontal lobe, which have found to be the most familiar location.

As illustrated in Table 2, 15 patients were less than 35 years of age, and 9 patients were older than 35 years. In the LAMA group, the majority of cases were male (71\%), and the most common presentation was symptoms of raised intracranial pressure (ICP) (54\%). Common sites for brain tumors in the LAMA group were the Frontal lobe followed by the parietal lobe accounted for $29 \%$ and $21 \%$, respectively. Nineteen patients $(79 \%)$ stayed in the hospital for less than one week. The most common reasons for singing LAMA were preferring treatment abroad (54\%) and refusing surgical intervention (25\%). Out of the 28 patients in the LAMA group, fifteen patients (63\%) lost to their follow up after signing LAMA, and eight patients (33\%) were treated abroad and attended for follow up with Neurosurgery Unit at $\mathrm{KH}$.

As illustrated in Table 2, 15 patients were less than 35 years of age, and nine patients were older than 35 years. The majority of LAMA cases were male (71\%), and the 
Table 1. Demographic Information of Included Patients With Brain Tumour Admitted to Khoula Hospital Between January 2017 to December 2018

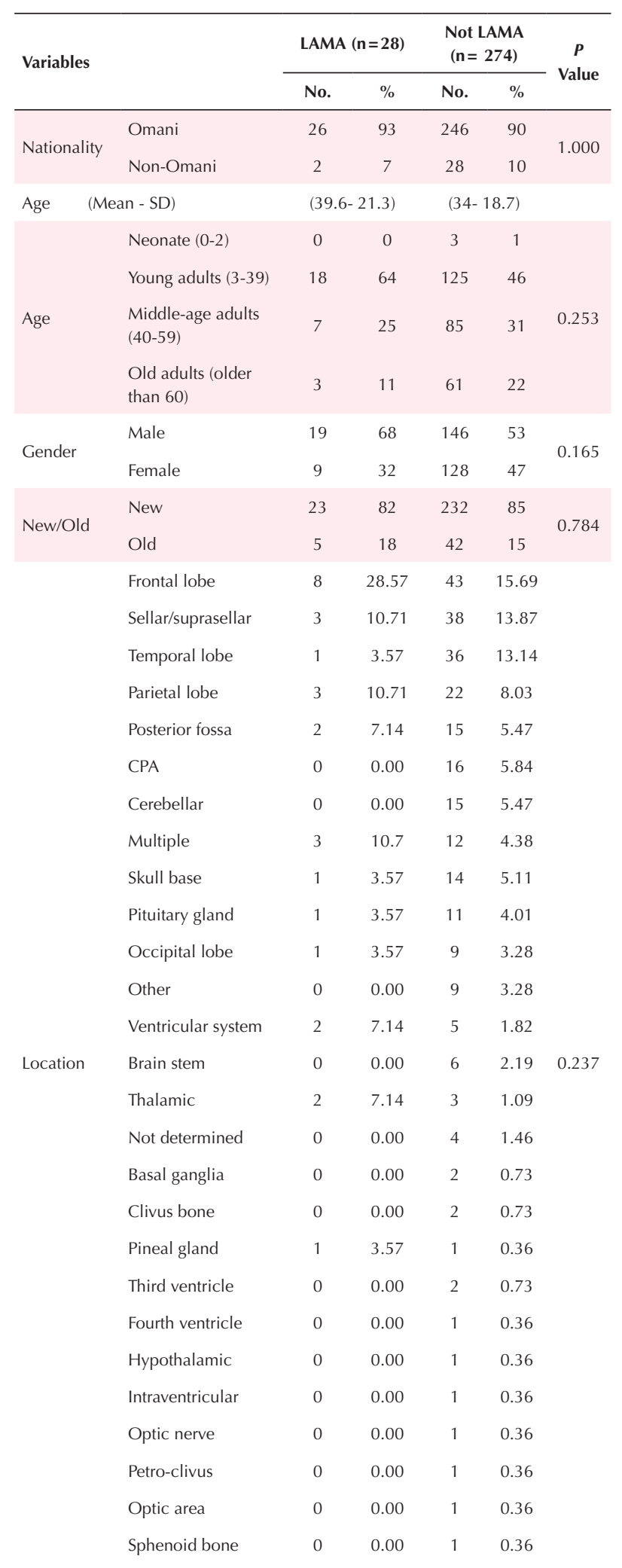

\begin{tabular}{|c|c|c|c|c|c|c|}
\hline \multirow{27}{*}{$\begin{array}{l}\text { Initial } \\
\text { diagnosis }\end{array}$} & Meningioma & 3 & 10.71 & 53 & 19.34 & \\
\hline & Pituitary adenoma & 1 & 3.57 & 37 & 13.50 & \\
\hline & Glioblastoma & 3 & 10.71 & 33 & 12.04 & \\
\hline & Not determined & 14 & 50.00 & 16 & 5.84 & \\
\hline & Metastasis & 1 & 3.57 & 27 & 9.85 & \\
\hline & Others & 0 & 0.00 & 19 & 6.93 & \\
\hline & Astrocytoma & 0 & 0.00 & 18 & 6.57 & \\
\hline & Oligodendroglioma & 2 & 7.14 & 11 & 4.01 & \\
\hline & Ependymoma & 0 & 0.00 & 8 & 2.92 & \\
\hline & $\begin{array}{l}\text { Glioma (not } \\
\text { determined) }\end{array}$ & 0 & 0.00 & 8 & 2.92 & \\
\hline & Schwannoma & 0 & 0.00 & 8 & 2.92 & \\
\hline & Craniopharyngioma & 1 & 3.57 & 6 & 2.19 & \\
\hline & Medulloblastoma & 1 & 3.57 & 6 & 2.19 & \\
\hline & Craniopharyngioma & 1 & 3.57 & 4 & 1.46 & 0.000 \\
\hline & Chordoma & 1 & 3.57 & 2 & 0.73 & \\
\hline & Lymphoma & 0 & 0.00 & 3 & 1.09 & \\
\hline & Neurocytoma & 0 & 0.00 & 3 & 1.09 & \\
\hline & Gangliocytoma & 0 & 0.00 & 2 & 0.73 & \\
\hline & Teratoid/rhabdoid & 0 & 0.00 & 2 & 0.73 & \\
\hline & $\begin{array}{l}\text { Anaplastic } \\
\text { oligodendroglioma }\end{array}$ & 0 & 0.00 & 1 & 0.36 & \\
\hline & B-cell lymphoma & 0 & 0.00 & 1 & 0.36 & \\
\hline & $\begin{array}{l}\text { Choroid plexuses } \\
\text { carcinoma }\end{array}$ & 0 & 0.00 & 1 & 0.36 & \\
\hline & Dermoid & 0 & 0.00 & 1 & 0.36 & \\
\hline & Ganglioglioma & 0 & 0.00 & 1 & 0.36 & \\
\hline & Ganglioma & 0 & 0.00 & 1 & 0.36 & \\
\hline & Germinoma & 0 & 0.00 & 1 & 0.36 & \\
\hline & Gliosarcoma & 0 & 0.00 & 1 & 0.36 & \\
\hline
\end{tabular}

Note. Location of the lesion is based on radiological report or operation notes.

SD: standard deviation, CPA: cerebellopontine angle

most common presentation was symptoms of raised ICP (54\%). The frontal lobe accounted for $29 \%$ of the LAMA cases, followed by the parietal lobe (21\%). Nineteen patients $(79 \%)$ stayed in the hospital for less than one week. The most common reasons for singing LAMA were preferring treatment abroad (54\%) and refusing surgical intervention (25\%). Fifteen patients (63\%) lost their follow up after signing LAMA, and eight patients (33\%) were treated abroad and came for follow up in the same hospital.

The association between the age and the treatment received shown in Table 3. There was no significant relationship between treatment type and age groups. There was no difference in the treatment outcome concerning the average age for both LAMA and nonLAMA group. Association of various variables with the frequency of LAMA and their relative significance are illustrated in Table 4 (age, gender, nationality, initial diagnosis, location, outcome, and prior LAMA) among LAMA cases by using Pearson $\chi^{2}$ test. The results revealed that there was a significant relationship between the reason for LAMA and gender $(P=0.020)$. However, there was no relationship between the reason for LAMA or 
Table 2. Analysis of LAMA Cases

\begin{tabular}{|c|c|c|c|}
\hline & & No. & $\%$ \\
\hline \multirow{2}{*}{ Age (y) } & Less than 35 & 15 & 63 \\
\hline & 35 and more & 9 & 38 \\
\hline \multirow{2}{*}{ Gender } & Male & 17 & 71 \\
\hline & Female & 7 & 29 \\
\hline \multirow{2}{*}{ Nationality } & Omani & 22 & 92 \\
\hline & Non-Omani & 2 & 8 \\
\hline \multirow{2}{*}{ Presenting complain } & Hight ICP symptoms & 13 & 54 \\
\hline & Convulsions & 11 & 46 \\
\hline \multirow{3}{*}{ Initial diagnosis } & Brain tumor & 19 & 79 \\
\hline & Stroke & 2 & 8 \\
\hline & Seizure disorders & 3 & 13 \\
\hline \multirow{9}{*}{ Location } & Frontal & 7 & 29 \\
\hline & Temporal & 1 & 4 \\
\hline & Parietal & 5 & 21 \\
\hline & Pituitary & 2 & 8 \\
\hline & Third ventricle & 2 & 8 \\
\hline & Thalamus & 2 & 8 \\
\hline & Pineal & 1 & 4 \\
\hline & Multiple & 2 & 8 \\
\hline & Other & 2 & 8 \\
\hline \multirow{2}{*}{ Length of hospital stay } & Less than one week & 19 & 79 \\
\hline & Week and more & 5 & 21 \\
\hline \multirow{4}{*}{ Reason for LAMA } & Refusing surgical intervention & 6 & 25 \\
\hline & Prefer treatment abroad & 13 & 54 \\
\hline & For family discussion & 3 & 13 \\
\hline & Not mentioned & 2 & 8 \\
\hline \multirow{3}{*}{ Outcome } & No follow up & 15 & 63 \\
\hline & $\begin{array}{l}\text { Received treatment abroad and } \\
\text { came for follow up }\end{array}$ & 8 & 33 \\
\hline & Re-admitted for treatment & 1 & 4 \\
\hline
\end{tabular}

ICP: intracranial pressure

length of hospital stay with the other variable.

\section{Discussion}

$\mathrm{KH}$ is one of the three tertiary hospitals in Oman, and it is considered a leading center in neurosurgical services at the national level, with around 1400 patients admitted per year, $10 \%$ of which diagnosed with brain and spinal tumors. ${ }^{9}$

A recent local audit conducted at $\mathrm{KH}$ found that $34 \%$ of the patients have not operated because they signed LAMA. It has found that patients who leave against medical advice require increased care resources as they often return with worsening clinical status. ${ }^{1}$ Thus a good knowledge of reasons for signing LAMA can improve the approach to such population. A cross-sectional study conducted at 4 hospitals in Iran found that $5.6 \%$ of all patients admitted to the Emergency Departments in one month self-discharged against medical advice. ${ }^{10}$ Another retrospective study carried out in pediatric wards at the Royal Hospital, Oman, found that out of 11482 regular discharges, there were 183 cases of LAMA (prevalence: $1.6 \%) .{ }^{11}$

Our study showed that LAMA incidence among patients with brain tumors was $\gg$ significant (9.2\%). Moreover, most of the patients were young adults (3-39 years) land males in both LAMA and non-LAMA groups. Most of the included cases newly diagnosed with $\gg$ the frontal lobe being the most common location $(84.4 \%$ and $16 \%$, respectively). About $18.5 \%$ of the studied cases diagnosed with meningiomas. $\mid$

Younger patients, male, Omani, newly diagnosed tumors, and tumors in the frontal lobe were all risk factors for LAMA. These findings regarding age and gender are in concordance with results from previous studies in the literature. ${ }^{12-16}$

The results showed that despite Omani patients receiving free treatment, they were still signing LAMA more than non-Omani, which lined with other studies in this region. This may be because of the presence of influence from society or relatives outside the Omanis hospital that might be lacking for those non-Omani. ${ }^{16}$

Our study found that the most common presentation of LAMA patients was symptoms of high ICP (headache, nausea, vomiting, and vision changes) followed by convulsions. Most of our patients who signed LAMA found to have tumors in the frontal lobe. However, there was no data from previous studies to compare these findings.

The length of hospital stay has consistently skewed toward shorter stays, with $79 \%$ of LAMA patients staying in the hospital for less than a week. ${ }^{12,14}$ On the contrary, other studies have found that more extended hospital stays

Table 3. AVONA Test Between Age and Treatment Received (Both LAMA and Non-LAMA Cases)

\begin{tabular}{lccccc}
\hline & Sum of Squares & $\boldsymbol{d f}$ & Mean Square & F & Sig. \\
\hline Between Groups & 774.236 & 1 & 774.236 & 1.741 & 0.188 \\
Within Groups & 133412.708 & 300 & 444.709 & & \\
Total & 134186.944 & 301 & & & \\
\hline
\end{tabular}

Table 4. Association Between Lengths of Hospital Stay and Reason of LAMA With Others Variables Among LAMA Cases

\begin{tabular}{lcc}
\hline Variables & $\begin{array}{c}\text { Length of Hospital } \\
\text { Stay }(\boldsymbol{P} \text { Value })\end{array}$ & $\begin{array}{c}\text { Reason for LAMA }(\boldsymbol{P} \\
\text { Value })\end{array}$ \\
\hline Age & 0.615 & 0.322 \\
Gender & 1.000 & 0.020 \\
Nationality & 0.380 & 0.605 \\
Presenting complain & 0.630 & 0.402 \\
Initial diagnosis & 0.667 & 0.908 \\
Location of the tumor & 0.817 & 0.317 \\
Reason for LAMA & 0.540 & - \\
Outcome & 0.838 & 0.302 \\
Prior LAMA & 1.000 & 0.823 \\
\hline
\end{tabular}


are more associated with LAMA since longer hospital stays increase the likelihood that patients with a strong desire to leave the hospital do so against medical advice. ${ }^{17}$ When looking into the reasons patients left against medical advice, we found that most preferred to receive their treatment abroad. This preference has supported by the results of a questionnaire study conducted by Al-Hinai et al in 2011, which showed that neurological diseases were the second most common type of disease in Omani patients traveling abroad for treatment. ${ }^{18}$ The reasons for medical travel by Omani patients suggested has attributed to long delays in public healthcare facilities, fewer competitive private facilities, the high cost of private medical services, and leisure facilities offered alongside healthcare treatments by some private facilities in some countries. ${ }^{19}$ This finding has not seen in other studies where reasons like personal and family reasons or dissatisfaction with the hospital surgeries were the main reasons behind signing LAMA. ${ }^{10,20}$

All patients who signed LAMA in our hospital have given follow up appointments. However, most of LAMA patients in our study lost to follow up after discharge. This is consistent with previous studies, which showed that these patients are at high risk for poor follow-up care for unresolved medical issues. In addition, selfdischarged patients are at high risk for readmissions with resultant longer hospital stays and, ultimately, higher costs of care. ${ }^{12}$ However, in our study, only $4 \%$ of patients were re-admitted after discharge, and this can be because patients went abroad for receiving treatment and came back for follow up in another neurosurgical institute here in Oman other than KH. In fact, many patients seek a second opinion or different consultations from other doctors in different hospitals until they get satisfied with the treatment plan.

We included age, gender, nationality, presenting complaint, initial diagnosis, location of CNS lesion, and LAMA's prior history of studying the different variables that may affect the decision to sign LAMA. Additional variables have observed to be involved in other similar studies such as length of hospital stay, insurance, and socioeconomic status, the hospital's region (urban vs. rural), marital status, and race. These variables are highly related to the background of each study cohort. For instance, insurance status can not apply in our study as the medical expenses in $\mathrm{KH}$ were covered by the government for Omani patients. Furthermore, a study conducted in Saudi Arabia reported that admission during the period of Eid Al-Adha (Muslim's Holiday) was significantly associated with an increased risk for discharge against medical advice, ${ }^{16}$ which highlights that not only demographic but also cultural factors should consider in the study of predictive factors for the decision of LAMA.

According to Kishore, younger age, male gender, people without medical insurance, low socioeconomic status, alcohol, and drug abuse, psychiatric problems form the risk-factors for LAMA. ${ }^{21}$ However, in our study cohort, the only gender was significantly associated with signing LAMA, and there was no association between LAMA and other pre-admission factors, including health insurance, distance from treating hospital, and co-existing comorbidities.

Following other literature, we found that the male gender is significantly associated with signing LAMA. ${ }^{15}$ However, a large study conducted in neurological patients in India reported that women are more at risk for LAMA. ${ }^{22}$ They have attributed this finding to the fact that their patients' characteristics are different from those described in previous literature.

Unlike the results of our study, several studies have concluded that younger age has correlated with a higher rate of LAMA. ${ }^{12,15,23}$ Similarly, shorter length of hospital stay and urban hospitals has reported associated with LAMA. $^{12}$

There are no clinical, regulatory, or professional standards that specify the designation of LAMA in most hospitals. However, physicians need to spend more time with the patient before signing LAMA. Better communication skills may influence the patient's decision making regarding leaving the hospital. Providing all available options to the patient is considered one of the patient's rights. Moreover, patients should be aware of the consequences of leaving the hospital against medical advice and the need to stay in the hospital for further investigation and treatment, especially with patients diagnosed with brain tumors. Hospitals need to create a protocol for dealing with such events. Make patients aware of the protocol from the time of admission or even at the emergency department . Monthly audits and regular follow-ups for patients who signed LAMA need to consider in our hospital.

\section{Limitations}

The study has conducted as a retrospective and in a single-center-more centers needed to be involved in the future with larger sample size. The follow up in our study was limited. Moreover, it was difficult to compare the current study's variable with previously published articles due to differences in the targeted population and the lack of similar articles in the literature.

\section{Conclusion}

The incidence of LAMA among patients with brain tumors was not negligible and presented a significant issue. Younger patients, male, Omani, newly diagnosed tumors, and tumors in the frontal lobe were all risk factors for LAMA. LAMA presents a challenging and concerning experience for physicians, and more education and awareness have recommended avoiding readmission and 
loss of follow up.

\section{Conflict of Interest}

None.

\section{Ethical Statement}

This study was approved by the Research Ethics Committee, Directorate General of Khoula Hospital, Ministry of Health, Muscat, Oman (Approval code: PROO 102019044).

\section{References}

1. Ding R, Jung JJ, Kirsch TD, Levy F, McCarthy ML. Uncompleted emergency department care: patients who leave against medical advice. Acad Emerg Med. 2007;14(10):870-6. doi: 10.1197/j.aem.2007.06.027.

2. McKinney PA. Brain tumours: incidence, survival, and aetiology. J Neurol Neurosurg Psychiatry. 2004;75 Suppl 2(Suppl 2):ii12-7. doi: 10.1136/jnnp.2004.040741.

3. World Health Organization (WHO). Key Statistics. WHO; 2020. [cited 30 June 2020]. Available from: https://www.who. int/cancer/resources/keyfacts/en/.

4. Ayed IA. What makes patients leave against medical advice? J Taibah Univ Med Sci. 2009:4(1):16-22. doi: 10.1016/S16583612(09)70077-0.

5. Ramakrishnan N, Ranganathan L, Abraham BK, Rajagopalan S, Venkataraman R. What happens to patients discharged against medical advice? Indian J Crit Care Med. 2018;22(8):580-4. doi: 10.4103/ijccm.IJCCM_101_18.

6. Alfandre DJ. "I'm going home": discharges against medical advice. Mayo Clin Proc. 2009;84(3):255-60. doi: 10.1016/ s0025-6196(11)61143-9.

7. Franks P, Meldrum S, Fiscella K. Discharges against medical advice: are race/ethnicity predictors? J Gen Intern Med. 2006;21(9):955-60. doi: 10.1111/j.1525-1497.2006.00505.x.

8. Brook M, Hilty DM, Liu W, Hu R, Frye MA. Discharge against medical advice from inpatient psychiatric treatment: a literature review. Psychiatr Serv. 2006;57(8):1192-8. doi: 10.1176/ps.2006.57.8.1192.

9. Mishra G, Al Saadi T, Salhotra N, Biniwale S, Hadad M, Hashim $M$ et al. Brain and spinal tumors incidence annual audit 2017 of Dept of Neurosurgery Khoula Hospital Muscat Oman: a review. Am J Med Case Rep. 2018;6(7):128-31. doi: 10.12691/ajmcr-6-7-2.

10. Noohi K, Komsari S, Nakhaee N, Yazdi Feyzabadi V. Reasons for discharge against medical advice: a case study of emergency departments in Iran. Int J Health Policy Manag. 2013;1(2):137-42. doi: 10.15171/ijhpm.2013.25.

11. Al-Ghafri M, Al-Bulushi A, Al-Qasmi A. Prevalence of and reasons for patients leaving against medical advice from paediatric wards in Oman. Sultan Qaboos Univ Med J. 2016;16(1):e74-7. doi: 10.18295/squmj.2016.16.01.013.

12. Ibrahim SA, Kwoh CK, Krishnan E. Factors associated with patients who leave acute-care hospitals against medical advice. Am J Public Health. 2007;97(12):2204-8. doi: 10.2105/ajph.2006.100164.

13. Lee CA, Cho JP, Choi SC, Kim HH, Park JO. Patients who leave the emergency department against medical advice. Clin Exp Emerg Med. 2016;3(2):88-94. doi: 10.15441/ceem.15.015.

14. Weingart SN, Davis RB, Phillips RS. Patients discharged against medical advice from a general medicine service. J Gen Intern Med. 1998;13(8):568-71. doi: 10.1046/j.15251497.1998.00169.x.

15. Seaborn Moyse H, Osmun WE. Discharges against medical advice: a community hospital's experience. Can J Rural Med. 2004;9(3):148-53.

16. Youssef A. Factors associated with discharge against medical advice in a Saudi teaching hospital. J Taibah Univ Med Sci. 2012;7(1):13-8. doi: 10.1016/j.jtumed.2012.07.006.

17. Hwang SW, Li J, Gupta R, Chien V, Martin RE. What happens to patients who leave hospital against medical advice? Cmaj. 2003;168(4):417-20.

18. Al-Hinai SS, Al-Busaidi AS, Al-Busaidi IH. Medical tourism abroad: a new challenge to Oman's health system-Al Dakhilya region experience. Sultan Qaboos Univ Med J. 2011;11(4):477-84.

19. Al-Hashar A, Al-Zakwani I. Omanis traveling abroad for healthcare: a time for reflection. Oman Med J. 2018;33(4):2712. doi: 10.5001/omj.2018.52.

20. Asadollah Pour Karimi SH, Mohseni Saravi B, Bagherian Farahabbadi E, Zamanfar D, Fallah M, Asadi Abokheily M. Studying the rate and causes of discharge against medical advice in hospitals affiliated to Mazandaran University of Medical Sciences. Mater Sociomed. 2014;26(3):203-7. doi: 10.5455/msm.2014.26.203-207.

21. Kishore B. Leave against medical advice: an universal phenomenon. Muller J Med Sci Res. 2013;4(1):1-2. doi: 10.4103/0975-9727.112258.

22. Lakhotia AN, Sodani A, Dube M, Telang K, Jain R, Athale S. Discharge against medical advice from neurology wards of a teaching hospital: A prospective observational study. Clin Epidemiol Glob Health. 2019;7(1):115-20. doi: 10.1016/j. cegh.2018.02.011.

23. El Sayed M, Jabbour E, Maatouk A, Bachir R, Dagher GA. Discharge against medical advice from the emergency department: results from a tertiary care hospital in Beirut, Lebanon. Medicine (Baltimore). 2016;95(6):e2788. doi: 10.1097/md.0000000000002788. 